\title{
Organic cation transporter 6 directly confers resistance to anticancer platinum drugs
}

\author{
TETSUYA OGURI, EIJI KUNII, SATOSHI FUKUDA, KAZUKI SONE, TAKEHIRO UEMURA, \\ OSAMU TAKAKUWA, YOSHIHIRO KANEMITSU, HIROTSUGU OHKUBO, \\ MASAYA TAKEMURA, KEN MAENO, YUTAKA ITO and AKIO NIIMI \\ Department of Respiratory Medicine, Allergy and Clinical Immunology, Nagoya City \\ University Graduate School of Medical Sciences, Nagoya 467-8601, Japan
}

Received June 14, 2016; Accepted September 28, 2016

DOI: $10.3892 /$ br.2016.772

\begin{abstract}
Organic cation transporters (OCTs) of the solute carrier family 22 have a critical role in the cellular uptake of anticancer platinum drugs. Recently, we found that a decreased OCT6 expression is associated with a reduced intracellular uptake of cisplatin (CDDP), and concomitant resistance to CDDP. In the present study, we examined whether OCTs directly confer resistance to another platinum drug, oxaliplatin (L-OHP). To address this, we used parental lung cancer cell lines, PC-14 and SBC3; L-OHP-resistant sublines, PC-14/L-OHP and SBC3/L-OHP; and one CDDP-resistant subline PC-14/CDDP, to examine the relationships between the expression of OCTs and intracellular platinum drug concentration or platinum drug resistance. The two L-OHP-resistant sublines showed cross resistance to CDDP and L-OHP, and a decreased expression of OCT6. The intracellular accumulation of L-OHP in PC-14/L-OHP cells was reduced compared with the parental cells. The findings suggested that a reduced OCT6 expression confers platinum drug resistance in the sublines by decreasing the uptake of platinum drugs. Using the PC-14/CDDP cell line engineered to overexpress OCT6, we confirmed that the intracellular L-OHP concentration was increased concomitantly with OCT6 overexpression compared with the parental cell line. Additionally, OCT6 was expressed in a screening panel of lung and colon cancer tissues and matched normal control tissues. Taken together with the previous results, the present findings indicate that OCT6 is directly involved in platinum drug resistance by mediating platinum drug uptake in cancer cells.
\end{abstract}

Correspondence to: Dr Tetsuya Oguri, Department of Respiratory Medicine, Allergy and Clinical Immunology, Nagoya City University Graduate School of Medical Sciences, 1 Kawasumi, Mizuho-cho, Mizuho-ku, Nagoya 467-8601, Japan

E-mail: t-oguri@med.nagoya-cu.ac.jp

Key words: organic cation transporter, cisplatin, oxaliplatin, drug resistance, multidrug resistance, toxin extrusion

\section{Introduction}

Although the development of molecular-targeted therapies has improved the prognoses for many types of cancer $(1,2)$, anticancer platinum drugs, such as cisplatin (CDDP) and oxaliplatin (L-OHP), remain key cancer chemotherapeutics (3). Nonetheless, the effectiveness of platinum drugs is limited by the development of resistance in many cases (3). Intracellular active drug concentration is very important for cytotoxicity in cancer cells (4). Additionally, various mechanisms, including drug inactivation by glutathione and overexpression of drug efflux pumps, facilitate platinum drug resistance (5-8).

Organic cation transporters (OCTs) are influx transporters belonging to the solute carrier family 22 (SLC22), which contains four subtypes, OCT1 (SLC22A1), OCT2 (SLC22A2), OCT3 (SLC22A3), and OCT6 (SLC22A16). OCTs have characteristic patterns of tissue expression and transportspecific substrates (9). Previously, OCTs were shown to play a critical role in the cellular uptake of platinum drugs in cancer cells (10). OCT1 and OCT2 are expressed in some colon cancer cell lines, and mediate the cellular uptake and cytotoxicity of L-OHP $(9,10)$. OCT2 transports CDDP, and is responsible for CDDP-induced nephrotoxicity in cancer patients (11). Recently, we found that a decreased OCT6 expression is associated with resistance to CDDP, mediated by a decreased intracellular uptake of the drug (12).

In the present study, we established L-OHP-resistant sublines to further investigate the relationship between the resistance to anticancer platinum drugs and OCTs.

\section{Materials and methods}

Cell lines and culture conditions. The parental lung adenocarcinoma cell line (PC-14) and CDDP-resistant (PC-14/CDDP) and L-OHP resistant (PC-14/L-OHP) derivatives, as well as the human small cell lung cancer cell line (SBC-3) and an L-OHP resistant derivative (SBC-3/L-OHP) were used in the present study. The L-OHP-resistant cells were created by continuous exposure to stepwise-increasing concentrations of L-OHP. Intermediate-resistant variants were cloned and cultured again in the presence of the maximum concentration of L-OHP at which they remained viable. The CDDP-resistant cell line, 
PC-14/CDDP, was engineered to overexpress SLC22A16, and was described previously (12). The cells were cultured in RPMI-1640 medium supplemented with $10 \%$ heat-inactivated FBS and $1 \%(\mathrm{v} / \mathrm{w})$ penicillin/streptomycin in a humidified chamber $\left(37^{\circ} \mathrm{C}, 5 \% \mathrm{CO}_{2}\right)$. CDDP and L-OHP were purchased from Nippon Kayaku (Tokyo, Japan).

Concentration of anticancer agents for $50 \%$ cell survival $\left(I C_{50}\right)$. Cells were cultured at $5 \times 10^{3}$ cells/well in 96-well tissue culture plates. To assess cell viability, stepwise 10 -fold dilutions of the anticancer drugs $(10-100 \mu \mathrm{M})$ were added $2 \mathrm{~h}$ after plating, and the cultures were incubated at $37^{\circ} \mathrm{C}$ for $96 \mathrm{~h}$. At the end of the culture period, $20 \mu \mathrm{l}$ of MTS [3-(4,5-dimethylthiazol-2-yl)-5-(3-carboxymethoxyphenyl-2-(4-sulfophenyl)-2H-tetrazolium] solution (CellTiter 96 ${ }^{\circledR}$ AQueous One Solution Cell Proliferation Assay, Promega, Madison, WI, USA) was added, the cells were incubated for a further $4 \mathrm{~h}$, and the absorbance was measured at $490 \mathrm{~nm}$ using an ELISA plate reader. Mean values were calculated from three independent experiments performed in quadruplicate. Chemosensitivity was expressed as the $\mathrm{IC}_{50}$ drug concentration, which was determined from the concentration-effect relationship by using Graph Pad Prism version 5.0 (GraphPad Software, La Jolla, CA, USA).

Protein extraction and western blotting. Equal amounts of total cell lysates were solubilized in sample buffer $(50 \mathrm{mM}$ Tris-HCl (pH 6.8), 2\% SDS, 1 mM EDTA, 10\% glycerol) with cOmplete ${ }^{\mathrm{TM}}$ Mini Protease Inhibitor Cocktail Tablets, and PhosSTOP Phosphatase Inhibitor Cocktail (Roche Applied Science, Mannheim, Germany). Subsequently, the lysates were electrophoresed on 7.5\% Ready Gel Tris- $\mathrm{HCl}$ Precast Gels (Bio-Rad Laboratories, Hercules, CA, USA) and transferred to Immobilon-P filters (Merck Millipore, Billerica, MA, USA). The filters were then incubated with primary antibodies for $2 \mathrm{~h}$ at room temperature, followed by horseradish peroxidase (HRP)-conjugated secondary antibodies for $1 \mathrm{~h}$. The following antibodies were used: anti-OCT1 (polyclonal antirabbit ab15112, 1:500), anti-OCT2 (monoclonal antirabbit ab33919, 1:10,000), anti-OCT3 (monoclonal antirabbit ab124826, 1:3,000), anti-multidrug and toxin extrusion 1 (MATE1) (polyclonal antirabbit ab104016, 1:500), anti-MATE2 (polyclonal antirabbit ab105050, 1:1,000) (these antidodies fromm Abcam, Cambridge, MA, USA), anti-OCT6 (polyclonal antigoat sc-132395, 1:1,000, Santa Cruz Biotechnology, Dallas, TX, USA), and HRP-conjugated secondary antibody (GE Healthcare Bio-Sciences, Pittsburgh, PA, USA). Anti-tubulin (monoclonal antimouse T6199, 1:10,000) and anti-vinculin antibodies (monoclonal antimouse V9131, 1:20,000) (Sigma Aldrich, St. Louis, MO, USA) were used as loading controls. The density of each visualized immunoreactive band associated with the protein expression levels was calculated using a densitometer relative to those associated with loading control bands. The Human Tumor/Normal Tissue Protein Lysate Assay Strip Arrays [ST1-6X-1 (lung cancer) and ST7-6X1 (colon cancer)] from Proteus BioSciences, Inc. (Ramona, CA, USA) were screened to examine OCT6 protein expression in human lung and colon cancer tissues, and their respective matched normal tissues using western blot analysis.
Cellular accumulation of platinum. PC-14 and PC-14/L-OHP cells $\left(1 \times 10^{7}\right)$ were incubated for 1 or $4 \mathrm{~h}$ at $37^{\circ} \mathrm{C}$ with 40 or $100 \mu \mathrm{M} \mathrm{L}$-OHP. After incubation, the cells were washed three times with ice-cold PBS and resuspended in $1.5 \mathrm{ml}$ PBS. After centrifugation for $5 \mathrm{~min}$ at $1,300 \mathrm{x} \mathrm{g}$, the cell pellets were homogenized and re-centrifuged for $10 \mathrm{~min}$ at 3,000 $\mathrm{x}$ g. The supernatants were collected and ultrafiltered using Centrifree tubes (Amicon Inc, Beverly, MA, USA). The platinum concentration in the supernatants was measured by an atomic absorption spectrometry assay.

Statistical analysis. Differences between the samples were evaluated for statistical significance using the Student's unpaired t-test. The level of significance was set at $5 \%$ with two-sided analysis.

\section{Results}

$I C_{50}$ values for $\mathrm{L}-\mathrm{OHP}$ and $\mathrm{CDDP}$ in $\mathrm{L}$-OHP-resistant cell lines. We measured the $\mathrm{IC}_{50}$ for $\mathrm{CDDP}$ and $\mathrm{L}-\mathrm{OHP}$ in the PC-14/L-OHP and SBC-3/L-OHP cell lines and their parental cells (Table I). The $\mathrm{IC}_{50}$ for L-OHP was 16.49- and 32.29-fold higher in the PC-14/L-OHP and SBC-3/L-OHP cells, respectively, compared with the parental cell lines. Similarly, the $\mathrm{IC}_{50}$ for CDDP was 4.69- and 2.26-fold higher in the PC-14/L-OHP and SBC-3/L-OHP cells, respectively, compared with the parental cell lines. The L-OHP-resistant cancer cells were cross-resistant to CDDP.

Protein expression levels of OCTs and MATE transporters in L-OHP-resistant cells. We examined the protein expression levels of OCTs and MATE proteins in parental and L-OHP-resistant cells (Fig. 1). Each of the visualized immunoreactive bands associated with the protein expression levels was calculated using a densitometer relative to those associated with the loading control bands. We found that the OCT6 protein expression levels in PC-14/L-OHP and SBC-3/L-OHP were downregulated compared with the parental cells, PC-14 and SBC-3. By contrast, OCT1 and OCT2 expression levels were similar in the parental and L-OHP-resistant cells, and OCT3 expression was not detected. The expression levels of MATE1 and MATE2 were not different in the parental and L-OHP-resistant cells.

Cellular accumulation of platinum. The intracellular accumulation of platinum was measured in PC-14 and PC-14/L-OHP cells to determine whether a lower expression of OCT6 in the drug-resistant cells resulted in a reduced influx of drugs. After treatment with 40 and $100 \mu \mathrm{M} \mathrm{L-OHP}$ over periods of 1 and $4 \mathrm{~h}$, the intracellular platinum accumulation was significantly lower in the PC-14/L-OHP cells compared with the parental PC-14 cells (Fig. 2).

Increased platinum accumulation in cells overexpressing OCT6. To further investigate the role of OCT6 in intracellular platinum drug accumulation, we used the previously established OCT6 overexpressing PC-14/CDDP cell line, which was engineered to constitutively express the OCT6 gene, $S L C 22 A 16$. The expression level of the OCT6 protein was markedly increased in OCT6 overexpressing PC-14/CDDP 
Table I. $\mathrm{IC}_{50}$ values in L-OHP-resistant cells and their parental cells.

\begin{tabular}{lccccrr}
\hline Cell line & $\begin{array}{c}\text { L-OHP } \\
\mathrm{IC}_{50}(\mu \mathrm{M})\end{array}$ & $95 \% \mathrm{CI}$ & $\mathrm{RR}$ & $\begin{array}{c}\mathrm{CDDP} \\
\mathrm{IC}_{50}(\mu \mathrm{M})\end{array}$ & $95 \% \mathrm{CI}$ & $\mathrm{RR}$ \\
\hline PC-14 & 0.68 & $0.45-1.04$ & & 9.43 & $5.56-16.00$ & \\
PC-14/L-OHP & 11.21 & $9.13-13.80$ & 16.49 & 44.22 & $24.20-84.20$ & 4.69 \\
SBC-3 & 0.07 & $0.03-0.14$ & & 2.92 & $1.88-4.53$ & 2.26 \\
SBC-3/L-OHP & 2.40 & $1.33-4.34$ & 32.29 & 6.60 & $4.76-9.51$ & \\
\hline
\end{tabular}

$\mathrm{RR}$, resistance rate: $\left(\mathrm{IC}_{50}\right.$ in resistant subline $) /\left(\mathrm{IC}_{50}\right.$ in the parental subline); L-OHP, oxaliplatin; CDDP, cisplatin; $\mathrm{SBC}-3$, human small cell lung cancer cell line; PC-14, parental lung adenocarcinoma cell line; CI, confidence interval.

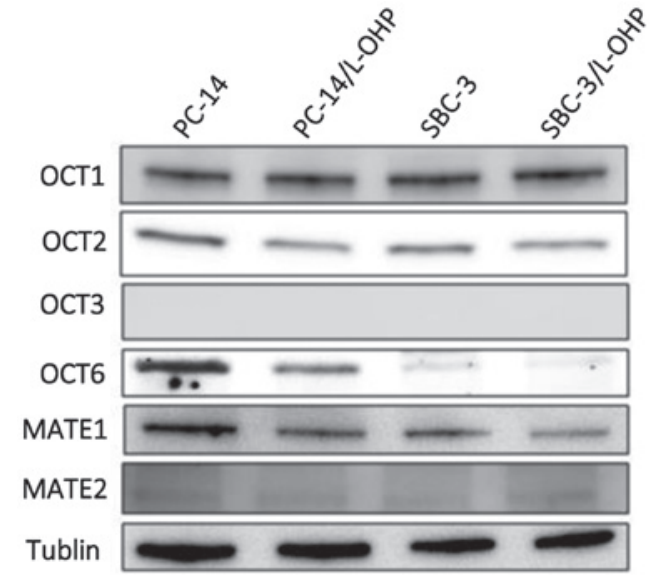

Figure 1. Protein expression levels of the OCTs and MATE transporters in lung cancer cell lines. The protein expression levels of OCT1, OCT2, OCT3, OCT6, MATE1, and MATE2 in the PC-14, PC-6/L-OHP, SBC-3, and SBC-3/L-OHP cells were assessed using western blotting. The levels of OCT6 protein in the PC-14/LOHP and SBC-3/L-OHP cells were downregulated compared with the parental cells, $\mathrm{PC}-14$ and SBC-3, respectively. OCTs, organic cation transporters; MATE, anti-multidrug and toxin extrusion; PC-14, parental lung adenocarcinoma cell line; L-OHP, oxaliplatin; SBC-3, human small cell lung cancer cell line.

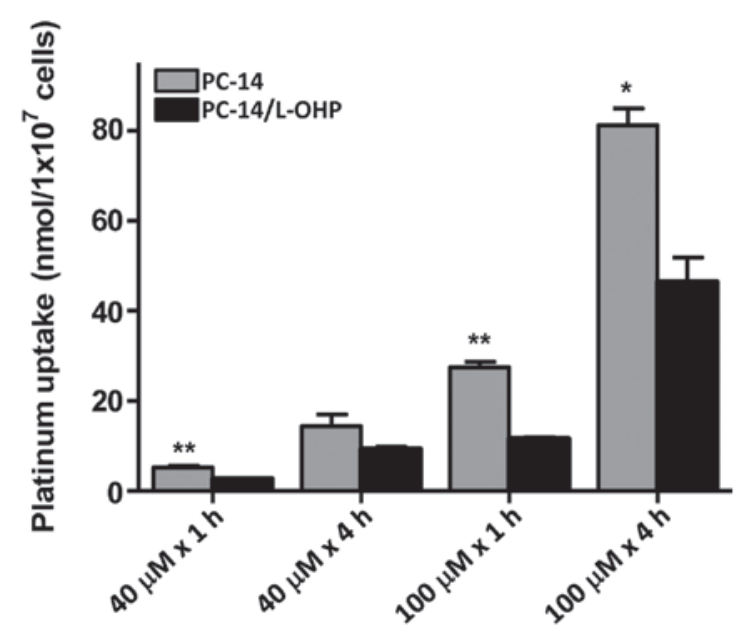

Figure 2. Intracellular platinum accumulation after L-OHP treatment. Intracellular platinum accumulation in PC-14 and PC-14/L-OHP cells after treatment with 40 or $100 \mu \mathrm{M}$ of L-OHP for 1 or $4 \mathrm{~h}$ was measured by an atomic absorption spectrometry assay. Results are expressed as the mean \pm standard deviation from three separate experiments. L-OHP, oxaliplatin; PC-14, parental lung adenocarcinoma cell line. ${ }^{* *} \mathrm{P}<0.005,{ }^{*} \mathrm{P}<0.05$, compared to the control. cells compared with the parental and mock-transfected cells (Fig. 3A). The intracellular platinum accumulation after treatment with $100 \mu \mathrm{M} \mathrm{L-OHP}$ for $4 \mathrm{~h}$ was significantly increased in the OCT6-overexpressing PC-14/CDDP cells compared with the mock-transfected control cells $(\mathrm{P}<0.05)$ (Fig. 3B).

OCT6 expression in human tissues. We investigated OCT6 protein expression in a panel of human lung and colon cancer tissue protein lysates, and their respective matched normal ones using western blot analysis. We found that OCT6 was detected in a screen panel of cancer and matched normal tissue protein lysates. In the lung cancers, the expression of OCT6 in cancer tissues was higher than that in the matched normal tissues. In the colon cancers, the expression of OCT6 was variable (Fig. 4).

\section{Discussion}

Recently, we reported that a decreased OCT6 expression is associated with resistance to CDDP, mediated by decreased intracellular uptake of the drug (12). In the present study, we established L-OHP-resistant cell lines and found that similar to CDDP, a decreased expression of OCT6 in these cells resulted in resistance to L-OHP, associated with the decreased intracellular uptake of L-OHP. Thus, OCT6 expression directly confers resistance to anticancer platinum drugs in cancer cells.

The efficacy of anticancer drugs in tumor cells is determined in part by the effective intracellular concentration of the drugs. Indeed, tissue platinum concentration is significantly associated with tumor response and survival in non-small cell lung cancer patients (13). Although OCTs play a critical role in the cellular uptake of platinum drugs, the relationship between OCTs and drug resistance is not fully understood. More specifically, although the characteristic substrate specificities for platinum drugs are known for some OCTs $(4,9)$, the specificities of OCT6 have not been comprehensively investigated. In this regard, the results from this, and our previous study, indicate that both CDDP and L-OHP are transported by OCT6. By contrast, the expression levels of OCT1, OCT2 and OCT3 were not altered in either CDDP or L-OHP resistant cells compared with the parental cells, suggesting that in this context, these OCT family members are not mainly involved in resistance to CDDP or L-OHP. 
A

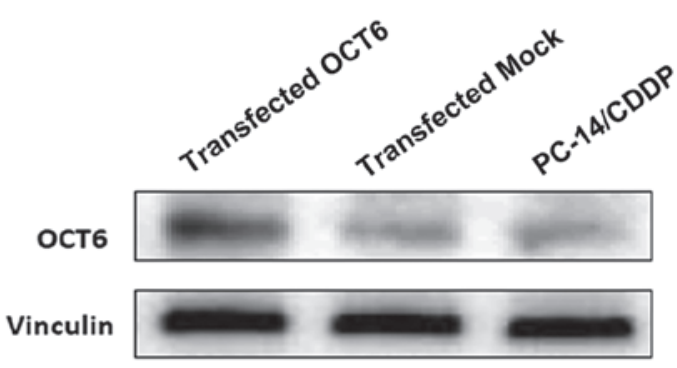

B

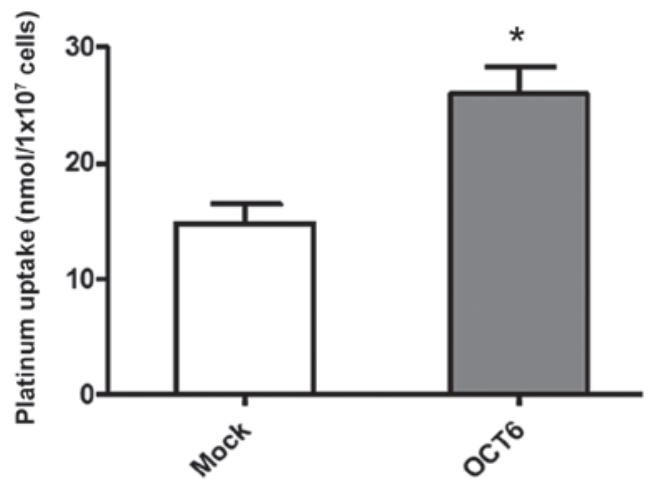

Figure 3. Effect of forced OCT6 expression on intracellular platinum accumulation. (A) Western blotting shows that OCT6 protein expression in PC-14/CDDP cells engineered to constitutively overexpress SLC22A16, is markedly increased compared with cells transfected with a control plasmid (mock) and parental cells (PC-14). (B) Intracellular platinum accumulation after treatment of PC-14/CDDP cells with $100 \mu \mathrm{M} \mathrm{L}-\mathrm{OHP}$ for $4 \mathrm{~h}$ compared with the control cells. Results are expressed as the mean \pm standard deviation from three separate experiments. ${ }^{*} \mathrm{P}<0.05$, compared to the control.

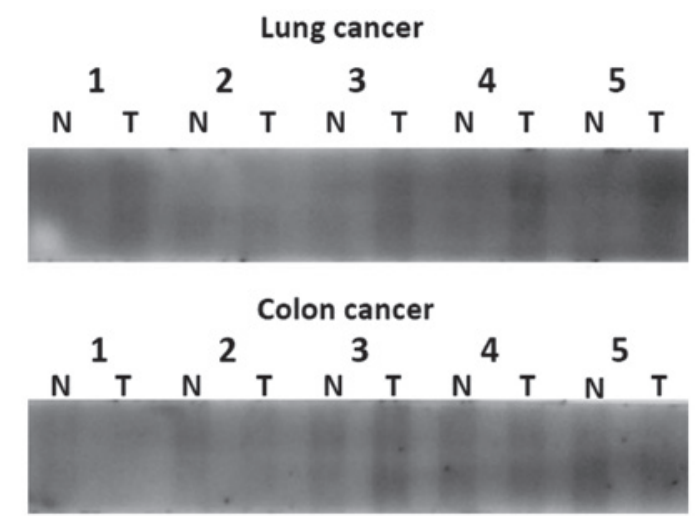

Figure 4. OCT6 expression in human tissues. OCT6 expression in human lung and colon cancer tissues, and their respective matched normal tissues assessed by western blotting. T, tumor tissue; N, normal tissue. Lung cancer: 1, Squamous cell carcinoma; 2, small cell carcinoma; 3, large cell carcinoma; 4, adenosquamous cell carcinoma; 5, adenocarcinoma. Colon cancer: Samples were classified histologically as adenocarcinoma.

Drug efflux systems are also considered to be another important determinant of the effective intracellular concentration of platinum drugs. Recently, the MATE multidrug efflux transporters were found to play crucial roles in platinum drug efflux from the kidney. L-OHP was transported intracellularly by OCT2, and MATE-mediated efflux of L-OHP from renal tubules protected against L-OHP nephrotoxicity. Notably, although CDDP was also transported by OCT2, MATE-mediated CDDP efflux was weak $(14,15)$. The pharmacological characteristics of L-OHP, which are different from those of CDDP, may also be a major reason for the low nephrotoxicity of L-OHP in the kidney. With these findings in mind, we investigated MATE1 and MATE2 expression in L-OHP-resistant lung cancer cells and found that expression of both did not differ between parental and L-OHP-resistant cells. We believe that these data are the first to indicate that MATEs expression is not a major determinant of resistance to platinum drugs in cancer cells.

A limitation of the present study was the exclusive use of lung cancer cell lines. Indeed, OCTs have characteristic patterns of tissue expression (9), and L-OHP is now mainly used in colon cancer chemotherapy (3). Previously, OCT1 and OCT2 were reported to be expressed in colon cancer samples, where OCT2 plays a critical role in the cellular uptake and consequent cytotoxicity of L-OHP $(9,10)$. In addition, recent clinical studies revealed that OCT2 expression is an important independent predictor of good outcome in 5-fluorouracil/leucovorin/L-OHP-treated metastatic colon cancers (16). OCT2 can also transport CDDP $(9,11)$, and OCT2 expression was reported to be a potential predictor of response to neoadjuvant chemotherapy with S-1/CDDP in gastric cancer (17). We detected OCT6 expression in primary colon cancer tissues and matched normal tissues, which is consistent with a previous report showing a strong expression of OCT6 in some colon and liver cancer cell lines (18). These data raise the possibility that OCT6 expression may influence the efficacy of L-OHP treatment for colon cancer.

The anticancer platinum drugs are necessary to improve survival even for those lung cancer patients treated with molecularly-targeted therapy (19). It may be noteworthy to determine whether the expression of OCT6 in lung cancer tissues, and potentially hematopoietic cells where OCT6 is also expressed (20), is an important predictor of efficacy and chemotoxicity after anticancer platinum drug chemotherapy.

Previous findings demonstrated the potential for drug-drug interactions when certain tyrosine kinase inhibitors are used concomitantly with drugs such as L-OHP, which are transported by OCTs (21). As the OCTs have an established role in regulating the pharmacokinetic profile of several anticancer drugs, as well as mediating the intrinsic cellular sensitivity of healthy organs and cancer cells, further studies are required to clarify the potential role of OCTs for reducing treatment related toxicity and improving the outcomes for cancer patients.

\section{Acknowledgements}

The present study was supported by a Grant-in-Aid from Kidani Memorial Trust and a Grant-in-Aid for Scientific Research (c) from the Japan Society for the Promotion of Science (MEXT/JSPSKAKENHI 15K08593).

\section{References}

1. Sculier JP, Berghmans T and Meert AP: Advances in target therapy in lung cancer. Eur Respir Rev 24: 23-29, 2015. 
2. Heinemann V, Douillard JY, Ducreux M and Peeters M: Targeted therapy in metastatic colorectal cancer - an example of personalised medicine in action. Cancer Treat Rev 39: 592-601, 2013

3. Kelland L: The resurgence of platinum-based cancer chemotherapy. Nat Rev Cancer 7: 573-584, 2007.

4. Dasari S and Tchounwou PB: Cisplatin in cancer therapy: Molecular mechanisms of action. Eur J Pharmacol 740: 364-378, 2014.

5. Oguri T, Fujiwara $\mathrm{Y}$, Isobe $\mathrm{T}$, Katoh $\mathrm{O}$, Watanabe $\mathrm{H}$ and Yamakido M: Expression of $\gamma$-glutamylcysteine synthetase $(\gamma$-GCS) and multidrug resistance-associated protein (MRP), but not human canalicular multispecific organic anion transporter (cMOAT), genes correlates with exposure of human lung cancers to platinum drugs. Br J Cancer 77: 1089-1096, 1998.

6. Oguri T, Fujiwara Y, Miyazaki M, Takahashi T, Kurata T, Yokozaki M, Ohashi $\mathrm{N}$, Isobe $\mathrm{T}$, Katoh $\mathrm{O}$ and Yamakido $\mathrm{M}$ : Induction of $\gamma$-glutamylcysteine synthetase gene expression by platinum drugs in peripheral mononuclear cells of lung cancer patients. Ann Oncol 10: 455-460, 1999.

7. Oguri T, Isobe T, Suzuki T, Nishio K, Fujiwara Y, Katoh O and Yamakido M: Increased expression of the MRP5 gene is associated with exposure to platinum drugs in lung cancer. Int J Cancer 86: 95-100, 2000

8. Oguri T, Isobe T, Fujitaka $\mathrm{K}$, Ishikawa $\mathrm{N}$ and Kohno $\mathrm{N}$ : Association between expression of the MRP3 gene and exposure to platinum drugs in lung cancer. Int J Cancer 93: 584-589, 2001.

9. Koepsell H, Lips K and Volk C: Polyspecific organic cation transporters: Structure, function, physiological roles, and biopharmaceutical implications. Pharm Res 24: 1227-1251, 2007.

10. Zhang S, Lovejoy KS, Shima JE, Lagpacan LL, Shu Y, Lapuk A, Chen Y, Komori T, Gray JW, Chen X, et al: Organic cation transporters are determinants of oxaliplatin cytotoxicity. Cancer Res 66: 8847-8857, 2006

11. Ciarimboli G, Ludwig T, Lang D, Pavenstädt H, Koepsell H, Piechota HJ, Haier J, Jaehde U, Zisowsky J and Schlatter E: Cisplatin nephrotoxicity is critically mediated via the human organic cation transporter 2. Am J Pathol 167: 1477-1484, 2005.

12. Kunii E, Oguri T, Kasai D, Ozasa H, Uemura T, Takakuwa O, Ohkubo H, Takemura M, Maeno K and Niimi A: Organic cation transporter OCT6 mediates cisplatin uptake and resistance to cisplatin in lung cancer. Cancer Chemother Pharmacol 75: 985-991, 2015.

13. Kim ES, Lee JJ, He G, Chow CW, Fujimoto J, Kalhor N, Swisher SG, Wistuba II, Stewart DJ and Siddik ZH: Tissue platinum concentration and tumor response in non-small-cell lung cancer. J Clin Oncol 30: 3345-3352, 2012.
14. Yokoo S, Yonezawa A, Masuda S, Fukatsu A, Katsura T and Inui K: Differential contribution of organic cation transporters, OCT2 and MATE1, in platinum agent-induced nephrotoxicity. Biochem Pharmacol 74: 477-487, 2007.

15. Yonezawa A and Inui K: Organic cation transporter OCT/SLC22A and $\mathrm{H}(+)$ /organic cation antiporter MATE/SLC47A are key molecules for nephrotoxicity of platinum agents. Biochem Pharmacol 81: 563-568, 2011

16. Tashiro A, Tatsumi S, Takeda R, Naka A, Matsuoka H, Hashimoto Y, Hatta K, Maeda K and Kamoshida S: High expression of organic anion transporter 2 and organic cation transporter 2 is an independent predictor of good outcomes in patients with metastatic colorectal cancer treated with FOLFOX-based chemotherapy. Am J Cancer Res 4: 528-536, 2014.

17. Naka A, Takeda R, Shintani M, Ogane N, Kameda Y, Aoyama T, Yoshikawa T, Kamoshida S and Kamoshida S: Organic cation transporter 2 for predicting cisplatin-based neoadjuvant chemotherapy response in gastric cancer. Am J Cancer Res 5: 2285-2293, 2015

18. Okabe M, Unno M, Harigae H, Kaku M, Okitsu Y, Sasaki T, Mizoi T, Shiiba K, Takanaga H, Terasaki T, et al: Characterization of the organic cation transporter SLC22A16: A doxorubicin importer. Biochem Biophys Res Commun 333: 754-762, 2005.

19. Zhou C, Wu YL, Chen G, Feng J, Liu XQ, Wang C, Zhang S, Wang J, Zhou S, Ren S, et al: Final overall survival results from a randomised, phase III study of erlotinib versus chemotherapy as first-line treatment of EGFR mutation-positive advanced non-small-cell lung cancer (OPTIMAL, CTONG-0802). Ann Oncol 26: 1877-1883, 2015.

20. Gong S, Lu X, Xu Y, Swiderski CF, Jordan CT and Moscow JA: Identification of OCT6 as a novel organic cation transporter preferentially expressed in hematopoietic cells and leukemias. Exp Hematol 30: 1162-1169, 2002.

21. Minematsu T and Giacomini KM: Interactions of tyrosine kinase inhibitors with organic cation transporters and multidrug and toxic compound extrusion proteins. Mol Cancer Ther 10: 531-539, 2011. 